\title{
Le potentiel fruitier de la forêt classée de Niangoloko au Burkina Faso
}

Paulin OUôBA*, Joseph BoussıM, Sita GuINKo

Laboratoire de Biologie et Écologie Végétales, Université de Ouagadougou, 03 BP 7021, Ouagadougou 03 ,

Burkina Faso

ouobapaulin@hotmail.com
* Correspondance et tirés à part

Reçu le 21 mars 2005 Accepté le 28 septembre 2005

Fruits, 2006, vol. 61, p. 71-81 (C) 2006 Cirad/EDP Sciences All rights reserved DOI: 10.1051/fruits:2006006

RESUMEN EsPañol, p. 81

\section{Fruit potential of the classified Niangoloko's forest in Burkina Faso.}

Abstract - Introduction. The aim of our study was to promote, in the classified Niangoloko's forest, other activities less destructive of the ecosystems than wood exploitation. There, four fruitbearing species (Detarium microcarpum Guill. \& Perr., Flacourtia flavescens Willd., Maranthes polyandra Benth. and Parinari curatellifolia Planch. ex Benth.) are abundantly represented. The pulp of each one of these four species is edible and can be processed into jam. Seeds of Maranthes polyandra and Parinari curatellifolia are oleaginous and are used in many sectors of industry. Unfortunately, if these fruits are used in the industry of other African countries, they are not known by the populations of Burkina Faso. Our study thus sought to constitute a database on the geographical distribution of the population of these fruit-bearing species, their ecology and their fruit production potential, in order to develop these resources better. Materials and methods. Using a soil map and a GPS, a map of the species associations could be realized. Stand inventory made it possible to measure the fruit-bearing tree species' density and height in each association. The average production of fruits per tree for each species was also measured. Results. Eleven species associations were listed and mapped. Each fruit-bearing species has a quite precise localization in one or more associations. Stands of D. microcarpum, F. flavescens, M. polyandra and $P$. curatellifolia were listed, with respective densities being able to reach $(1486 \pm 110 ; 160 \pm 33$; $313 \pm 32$ and $392 \pm 39)$ trees $\cdot \mathrm{ha}^{-1}$. The fruit quantity produced per individual was, respectively, $(2.1 \pm$ $0.6 ; 0.5 \pm 0.2 ; 0.4 \pm 0.2$ and $1.0 \pm 0.4) \mathrm{kg}$ of dry fruits. The average height of the fruit trees hardly exceeds $(3.7 \pm 0.3) \mathrm{m}$, which allows easy picking of the fruits without risk of accidents. Conclusion. The ecological conditions of the forest allow commercial exploitation of the fruits. The fruit-bearing potential of the classified Niangoloko's forest is very significant and presents interesting financial prospects for the riparian populations.

Burkina Faso / Detarium microcarpum / Flacourtia flavescens / Maranthes polyandra / Parinari curatellifolia / forests / forest stands / fruit crops / forest inventories / resource management

\section{Le potentiel fruitier de la forêt classée de Niangoloko au Burkina Faso.}

Résumé - Introduction. L'objectif de notre étude a été de promouvoir, dans la forêt classée de Niangoloko, d'autres activités moins destructrices des écosystèmes que l'exploitation du bois. Quatre espèces fruitières (Detarium microcarpum Guill. \& Perr., Flacourtia flavescens Willd., Maranthes polyandra Benth. et Parinari curatellifolia Planch. ex Benth.) y sont abondamment réprésentées. La pulpe de chacune de ces quatre espèces est comestible et peut être transformée en confiture. Les graines de Parinari curatellifolia et Maranthes polyandra sont oléagineuses et sont utilisées dans de nombreux secteurs de l'industrie. Malheureusement, si ces fruits sont utilisés dans l'industrie d'autres pays africains, ils ne sont pas connus des populations du Burkina Faso. Notre étude a donc cherché à constituer une base de données sur la localisation des peuplements d'espèces fruitières, leur écologie et leur potentiel de production en fruits afin de mieux valoriser ces ressources. Matériel et méthode. À l'aide d'une carte pédologique et d'un GPS, une carte des groupements végétaux a pu être réalisée. Des relevés ont permis de mesurer la densité et la hauteur des arbres des espèces fruitières dans chaque groupement. La production moyenne de fruits par arbre pour chaque espèce a également été mesurée. Résultats. Onze groupements végétaux ont été recensés et cartographiés. Chaque espèce fruitière a une localisation bien précise dans un ou plusieurs groupements. Des peuplements de Detarium microcarpum, Flacourtia flavescens, Maranthes polyandra et Parinari curatellifolia ont été recensés avec des densités respectives pouvant atteindre $(1486 \pm 110 ; 160 \pm 33 ; 313 \pm 32$ et $392 \pm 39)$ individus $\mathrm{ha}^{-1}$. La quantité de fruits produite par individu a été respectivement de $(2,1 \pm 0,6 ; 0,5 \pm 0,2 ; 0,4 \pm 0,2$ et $1,0 \pm 0,4) \mathrm{kg}$ de fruits secs. La hauteur moyenne des arbres fruitiers ne dépasse guère $(3,7 \pm 0,3) \mathrm{m}$, cela permet une collecte facile des fruits sans risque d'accidents. Conclusion. Les conditions écologiques permettent une exploitation commerciale des fruits. Le potentiel fruitier de la forêt classée de Niangoloko est très important et présente d'intéressantes perspectives financières pour les populations riveraines.

Burkina Faso / Detarium microcarpum / Flacourtia flavescens / Marantbes polyandra / Parinari curatellifolia / forêt / peuplement forestier / plante fruitière / inventaire forestier / gestion des ressources 


\section{Introduction}

Les formations végétales de l'Afrique tropicale se dégradent à des rythmes inquiétants. L'homme a toujours été cité comme le principal facteur de cette dégradation [1, 2]. Jusqu'alors, les réserves naturelles et les parcs nationaux étaient devenus un atout majeur pour la conservation de la biodiversité [3] parce qu'ils étaient relativement bien protégés. Malheureusement, du fait de l'explosion démographique et de la paupérisation croissante des populations, ces aires protégées sont aujourd'hui convoitées par les populations riveraines qui, très souvent, violent ces domaines à la recherche de nouvelles terres pour l'agriculture ou d'aires de coupes pour le bois de chauffe. Si des mesures conservatoires ne sont pas prises pour une gestion durable des ressources naturelles de ces forêts classées, il risque de se produire, dans bien des régions d'Afrique, une déforestation importante pouvant conduire à une perte considérable de la biodiversité. L'objectif de notre étude a été de promouvoir, dans la forêt classée de Niangoloko, d'autres activités moins destructrices des écosystèmes que l'exploitation du bois. La commercialisation de produits forestiers encore inexploités, autres que le bois, pourrait être l'une de ces activités à développer.

Dans la forêt classée de Niangoloko, plusieurs espèces fruitières se développent spontanément et en peuplements. Parmi elles, quatre espèces sont particulièrement abondantes : Detarium microcarpum, Flacourtia flavescens, Parinari curatellifolia et Maranthes polyandra. La pulpe de chacun des fruits de ces quatre espèces est comestible et peut être transformée en confiture. Les graines de Parinari curatellifolia sont oléagineuses; l'huile extraite des graines est utilisée dans de nombreux secteurs de l'économie [4]. Les graines de Maranthes polyandra sont également oléagineuses [5] et l'huile extraite pourrait intervenir dans la fabrication du savon. Les graines de Detarium microcarpum sont utilisées en pâtisserie [5].

La forêt classée de Niangoloko est ainsi riche en ressources fruitières à haute valeur économique. Malheureusement, ce fort potentiel reste inexploité et ne donne lieu qu'à une simple exploitation de cueillette pour une consommation locale. L'usage industriel des fruits de ces espèces, bien connus dans certains pays africains, n'est cependant pas connu au Burkina Faso. L'exploitation fruitière pourrait dès lors présenter des perspectives économiques intéressantes pour les populations riveraines de la forêt classée si ces produits fruitiers étaient valorisés. Cela pourrait être un palliatif à la coupe abusive dont font l'objet les arbres de la forêt de Niangoloko. Notre étude a donc cherché à constituer une base de données sur la localisation des peuplements d'espèces fruitières, leur écologie et leur potentiel de production en fruits afin de mieux exploiter ces ressources fruitières.

\section{Matériel et méthodes}

\subsection{La zone d'étude}

La forêt classée de Niangoloko appartient au domaine forestier classé de l'État depuis 1936. Elle est située au sud-ouest du Burkina Faso (figure 1) et s'étend sur une superficie de 7295,83 ha entre $4^{\circ} 50^{\prime}$ et $4^{\circ} 58^{\prime}$ de longitude $\mathrm{O}$ et entre $10^{\circ} 10^{\prime}$ et $10^{\circ} 17^{\prime}$ de latitude N. Tout autour de la forêt se sont installés des villages d'ethnies Goin (agriculteurs) et Peulh (éleveurs). Les principales activités autorisées au sein de la forêt sont la cueillette des fruits, l'exploitation du miel, le prélèvement des plantes médicinales et le ramassage du bois mort.

Le climat est de type soudano-guinéen. Selon les données de la Direction de la météorologie nationale, la pluviométrie moyenne de 1980 à 2000 à Banfora a été de $1034 \mathrm{~mm} \cdot \mathrm{an}^{-1}$. Les pluies s'installent à partir du mois de mai et se poursuivent jusqu'en octobre avec néanmoins la possibilité de quelques pluies dès le mois de février; les températures moyennes varient peu : de (25 à 31$)^{\circ} \mathrm{C}$.

La couverture géologique est constituée de granites d'âge indéterminé [6]. La géomorphologie est caractérisée par une succession de croupes à sommets convexes ou plats. De ces croupes s'étirent de longs glacis de raccordement dont les pentes varient entre (1 et 3) \% [6].

Le réseau hydrographique est composé de nombreux cours d'eau à écoulement 
temporaire (figure 2). De nos jours, seul le sol a fait l'objet d'études approfondies, cela a permis de caractériser les propriétés physiques et chimiques des différentes unités de sols de cette forêt et de réaliser une carte des unités pédologiques (figure 2). Trois grandes classes de sols ont été recensées : les lithosols sur cuirasses ferrugineuses, les sols ferrugineux tropicaux lessivés et les sols hydromorphes [6].

La végétation caractéristique est une mosaïque de forêts-galeries, de savanes arbustives, de savane arborée et de savanes boisées. De nombreuses espèces figurant sur la liste des espèces intégralement protégées ou menacées de disparition dans les autres contrées du pays y sont toujours bien représentées. Ce sont entre autres : Adansonia digitata $\mathrm{L}$., Anogeissus leiocarpa (D C.) Guill \& Perr., Khaya senegalensis (Desr.) A. Juss., Parkia biglobosa (Jacq.) Benth., Detarium microcarpum Guill. \& Perr., Diospyros mespiliformis Hochst. ex A. Rich., Entada africana Guill. \& Perr., Zanthoxylum xanthoxyloides (Lam.) Watermann, Sclerocarya birrea (A. Rich.) Hochst., Saba senegalensis (A. DC.) Pichon et Ximenia americana L. [7].

\subsection{Méthodes d'étude}

La méthode utilisée a eu pour objectif principal de localiser et de cartographier les peuplements d'arbres fruitiers de la forêt classée de Niangoloko pour faciliter leur accès par ceux qui auront en charge la collecte des fruits. Un second objectif a été défini, celui de dénombrer les arbres fruitiers et de mesurer la production fruitière des individus de chaque espèce.

Pour localiser et cartographier les peuplements d'arbres fruitiers, une subdivision de la végétation de la forêt classée en ses différentes unités de végétations appelées groupements végétaux a été réalisée. Chaque groupement végétal a été nommé par l'espèce dominante. Pour rechercher les différents groupements végétaux (figure 2), une carte pédologique à grande échelle (1/ 20000) [6] a été utilisée pour faire la prospection. Les coordonnées géographiques de toutes les unités de sol ont été enregistrées avec un GPS (Global Positioning System) et, sur le terrain, les unités de sol ont été une

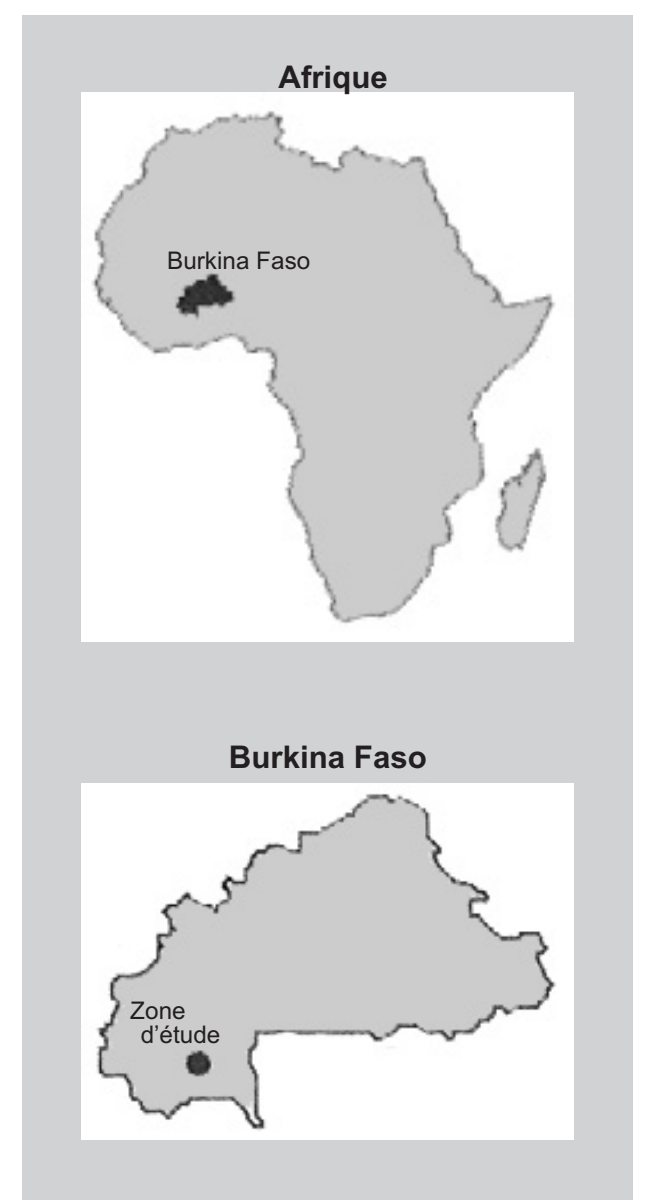

Figure 1.

Localisation de la forêt classée de Niangoloko (Burkina Faso). à une parcourues afin de décrire le type de groupement qui les recouvrait. Les contours de chaque groupement identifié sur un type de sol donné ont été délimités à l'aide de points GPS. Les logiciels Arc-view 3.2 et Arcinfo 3.2 ont permis d'utiliser ces coordonnées GPS pour réaliser une carte des groupements. Celle-ci étant faite, nous avons pu obtenir la superficie de chaque groupement en utilisant le même logiciel Arc-view.

Un inventaire forestier a ensuite permis de connaître la densité de chaque espèce dans chaque groupement ; cinq à dix relevés ont été effectués par groupement identifié, selon son étendue. La surface des relevés a été de $600 \mathrm{~m}^{2}(30 \mathrm{~m} \times 20 \mathrm{~m})$. Les relevés ont été faits pendant la période de floraison ou de fructification de chaque espèce pour compter les individus en âge de fructifier. 


\section{P. Ouôba et al.}

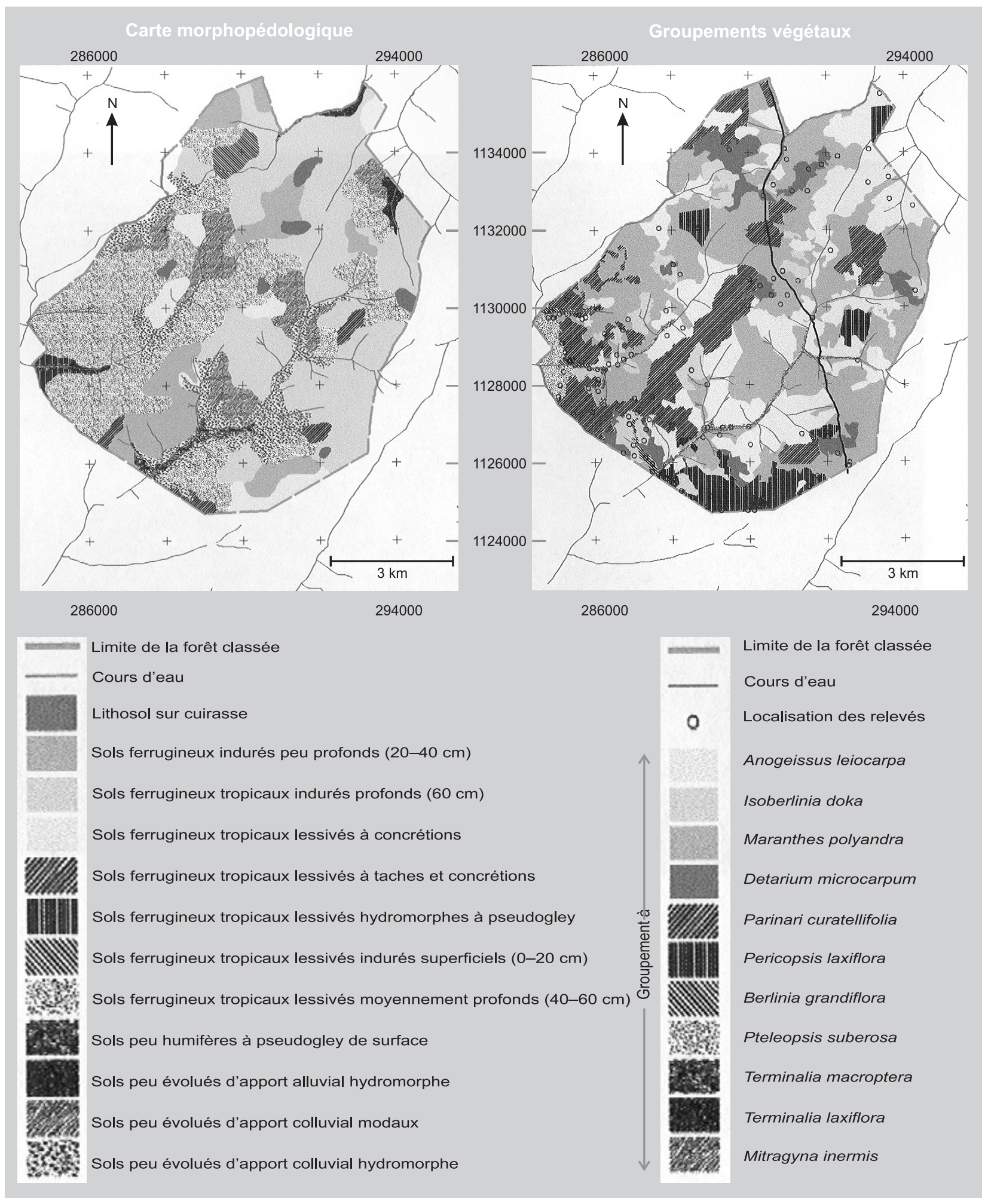

Figure 2.

Cartes des sols et des groupements végétaux de la forêt classée de Niangoloko (Burkina Faso). 
Tableau I.

Groupements végétaux identifiés dans la forêt classée de Niangoloko, répartis selon la topographie et la nature du sol (Burkina Faso).

\begin{tabular}{|c|c|c|c|}
\hline Groupement & Sols & Topographie & $\begin{array}{l}\text { Superficie } \\
\text { (ha) }\end{array}$ \\
\hline Anogeissus leiocarpa & $\begin{array}{l}\text { Sols peu évolués d'apports alluvial ou colluvial hydromorphe } \\
\text { ou d'apport colluvial modaux }\end{array}$ & Bas de pente & 1916 \\
\hline Berlinia grandiflora & Sols peu humifères à pseudogley de surface & Fond de vallée & 43 \\
\hline Detarium microcarpum & Lithosols sur cuirasse et sols ferrugineux tropicaux indurés superficiels & Haut glacis & 402 \\
\hline Isoberlinia doka & Lithosols sur cuirasse et sols ferrugineux tropicaux indurés superficiels & Haut glacis & 391 \\
\hline Maranthes polyandra & Sols ferrugineux tropicaux indurés profonds & Pente moyenne & 2777 \\
\hline Mitragyna inermis & Sols peu humifères à pseudogley de surface & Fond de vallée & 78 \\
\hline Parinari curatellifolia & Sols ferrugineux tropicaux indurés peu et moyennement profonds & Pente moyenne & 1098 \\
\hline Pericopsis laxiflora & $\begin{array}{l}\text { Sols ferrugineux tropicaux lessivés à concrétions } \\
\text { ou à taches et concrétions }\end{array}$ & Pente moyenne & 431 \\
\hline Pteleopsis suberosa & $\begin{array}{l}\text { Sols ferrugineux tropicaux lessivés hydromorphes } \\
\text { à pseudogley }\end{array}$ & Bas de pente & 124 \\
\hline Terminalia laxiflora & Sols peu humifères à pseudogley de surface & Fond de vallée & 25 \\
\hline Terminalia macroptera & Sols peu humifères à pseudogley de surface & Fond de vallée & 10 \\
\hline
\end{tabular}

La hauteur de tous les individus en âge de fructifier a été notée également afin de calculer la hauteur moyenne des individus de chaque espèce fruitière. Ce paramètre est très important pour évaluer l'effort à fournir pour accéder aux fruits et les risques d'accident lors des récoltes. Pour Terborgh ${ }^{1}$, la difficulté pour obtenir des mesures précises de la quantité de fruits produits par une forêt tropicale est due au fait que presque tous les fruits sont produits en hauteur dans la canopée où ils sont alors inaccessibles aux hommes.

La production moyenne par arbre pour chaque espèce a également été mesurée. Dix arbres fruitiers ont été sélectionnés de manière aléatoire par espèce : pour cela dix points ont été choisis au hasard dans les peuplements d'arbres fruitiers, puis repérés sur le terrain à l'aide d'un GPS ; l'arbre fruitier le plus proche du point sélectionné a alors été retenu pour l'échantillonnage. Cette méthode, dite méthode des distances, est fréquemment utilisée pour l'échantillon-

\footnotetext{
${ }^{1}$ Voir Terborgh J., Les ressources végétales clés de la forêt tropicale, 1999, http:// carpe.umd.edu/products/PDF_files/ReportTerborgh.pdf.
}

nage en forêts [8]. Après repérage de l'arbre fruitier retenu, tous ses fruits ont alors été cueillis, comptés et un échantillonnage de dix fruits a été prélevé pour être séché et pesé afin d'obtenir le poids moyen des fruits secs. Cette collecte a eu lieu à la période de maturité de chaque espèce fruitière afin de ne compter que les fruits parvenant à maturité.

Pour obtenir le poids de fruits produit par un individu, le nombre moyen de fruits par individu pour une espèce donnée a été multiplié par le poids moyen du fruit de l'espèce.

\section{Résultats}

\subsection{Localisation des groupements végétaux de la forêt classée}

Onze groupements végétaux ont été recensés dans la forêt classée de Niangoloko (figure 2) : les groupements à Anogeissus leiocarpa, Isoberlinia doka Craib et Stapf, Maranthes polyandra, Detarium microcarpum, Parinari curatellifolia, Pericopsis laxiflora (Benth. ex Back.) Van Meeuwen, Berlinia grandiflora (Vahl) Hutch. et Dalz., Pteleopsis suberosa Engl. \& Diels, Terminalia macroptera Guill. et Perr., Terminalia 
Tableau II.

Densité et hauteur moyenne des individus étudiés dans les groupements végétaux de la forêt classée de Niangoloko (Burkina Faso).

\begin{tabular}{lcccc} 
Groupement & \multicolumn{2}{c}{$\begin{array}{c}\text { Densité des individus dans les différents groupements } \\
\text { (arbres·ha }{ }^{-1} \text { ) }\end{array}$} \\
& D. microcarpum & F. flavescens & M. polyandra & P. curatellifolia \\
\hline Anogeissus leiocarpa & $29 \pm 16$ & $154 \pm 24$ & 0 & 0 \\
Detarium microcarpum & $1486 \pm 110$ & 0 & 0 & $75 \pm 16$ \\
Maranthes polyandra & 0 & $160 \pm 33$ & $313 \pm 32$ & $105 \pm 17$ \\
Parinari curatellifolia & 0 & 0 & $39 \pm 18$ & $392 \pm 39$ \\
Pericopsis laxiflora & $70 \pm 20$ & 0 & $120 \pm 23$ & 0 \\
Berlinia grandiflora, Isoberlinia doka, & 0 & & 0 & 0 \\
Mitragyna inermis, Pteleopsis suberosa, & & & 0 \\
Terminalia laxiflora, Terminalia macroptera & & &
\end{tabular}

\begin{tabular}{lc} 
Groupement & $\begin{array}{c}\text { Hauteur moyenne des individus } \\
(\mathrm{m})\end{array}$ \\
\hline D. microcarpum & $3,7 \pm 0,4$ \\
F. flavescens & $3,0 \pm 0,1$ \\
M. polyandra & $3,7 \pm 0,3$ \\
P. curatellifolia & $3,2 \pm 0,2$
\end{tabular}

laxiflora Engl. et Mitragyna inermis (Willd.) O. Ktze.

Une liaison entre les différents milieux écologiques (topographie et sols) et les différents groupements identifiés a été observée (figure 2 , tableau I) :

Les caractéristiques physico-chimiques des sols telles que la profondeur, la réserve en eau et le $\mathrm{pH}$ au sein des onze groupements végétaux ont été évaluées (figure 3). Trois groupements supportent de larges variations de $\mathrm{pH}$. Ce sont les groupements à Pericopsis laxiflora, Anogeissus leiocarpa, et Maranthes polyandra. Les quatre groupements Maranthes polyandra, Parinari curatellifolia, Isoberlinia doka et Detarium microcarpum supportent des sols peu profonds et des réserves en eau négligeables.

\subsection{Localisation des espèces fruitières}

Parmi les onze groupements recensés dans la forêt classée de Niangoloko, six groupements végétaux ne contiennent pas les individus des quatre espèces fruitières Detarium microcarpum, Flacourtia flavescens, Parinari curatellifolia et Maranthes polyandra.
Ce sont les groupements à Berlinia grandiflora, Isoberlinia doka, Mitragyna inermis, Pteleopsis suberosa, Terminalia laxiflora, et Terminalia macroptera (tableau II). Ces groupements sont de superficies très réduites.

Parmi les quatre espèces fruitières dominantes répertoriées, trois espèces sont abondantes et dominantes dans trois groupements si bien qu'elles donnent chacune son nom au groupement correspondant. C'est le cas de Detarium microcarpum, Maranthes polyandra et Parinari curatellifolia (tableau II). L'espèce Flacourtia flavescens se trouve localisée dans deux groupements à savoir les groupements à Anogeissus leiocarpa et à Maranthes polyandra. Les espèces fruitières Detarium microcarpum, Flacourtia flavescens et Maranthes polyandra sont localisées dans les trois groupements qui supportent de larges variations de $\mathrm{pH}$. Ce sont les groupements à Anogessus leiocarpa, Maranthes polyandra et Pericopsis laxiflora (figure 3). Ces trois espèces fruitières ne sont donc pas très exigeantes en termes de conditions chimiques du sol et elles peuvent donc évoluer sur une gamme variée de sols. De plus, les individus des quatre espèces fruitières principales sont localisés dans les groupements qui colonisent des sols peu profonds et des réserves en eau négligeables (figure 3). Ce sont les groupements à Detarium microcarpum, Maranthes polyandra et Parinari curatellifolia. Ce qui implique que ces quatre espèces fruitières peuvent supporter un stress hydrique plus ou moins important. 
Enfin, la hauteur moyenne des individus de chacune des quatre espèces ne dépasse guère 3,7 m (tableau II) ; cela permet une collecte rapide et facile des fruits sans risque d'accident.

\subsection{Le potentiel fruitier}

Le potentiel fruitier de la forêt classée de Niangoloko est immense ; avec des densités respectives de $(1486 \pm 110,313 \pm 32$ et $392 \pm$ 39) individus $\cdot \mathrm{ha}^{-1}$, les peuplements à Detarium microcarpum, Maranthes polyandra et Parinari curatellifolia couvrent d'importantes superficies, respectivement 402, 2777 et 1098 ha (sur une superficie totale de 7300 ha) (tableau I). L'espèce fruitière Flacourtia flavescens se rencontre abondamment dans deux groupements, à savoir les groupements à Anogeissus leiocarpa et à Maranthes polyandra (tableau II). Cette espèce fruitière n'est pas dominante dans ces groupements, mais elle s'y trouve avec des densités importantes, respectivement $(160 \pm 33$ et $154 \pm 24)$ individus ha $^{-1}$; en termes de superficie, Flacourtia flavescens couvre la superficie la plus importante avec un cumul de 4693 ha du fait qu'elle se retrouve en fortes densités dans les deux groupements les plus étendus de la forêt, à savoir les groupements à Anogeissus leiocarpa et à Maranthes polyandra ; cela représente plus de la moitié de la superficie de la forêt classée (tableau I).

Le nombre de fruits produits par individu varie de $130 \pm 64$ à $435 \pm 122$ en fonction des espèces (tableau III). Parmi les quatre espèces considérées, ce sont les arbres de Flacourtia flavescens qui produisent le plus de fruits. La production moyenne annuelle en fruits, évaluée en poids sec, est la plus élevée pour les individus de Detarium microcarpum chez qui elle atteint $(2,1 \pm$ $0,6) \mathrm{kg}$ (tableau III). Néanmoins, au sein de chacune des quatre espèces, il apparaît une variation souvent importante de la productivité d'un individu à l'autre.

Quelle que soit l'espèce fruitière considérée, la date de maturité et de récolte des fruits peut s'étaler sur plusieurs mois (tableau III) : les fruits de Detarium microcarpum sont disponibles de février à avril ; ceux de Flacourtia flavescens, de juillet à

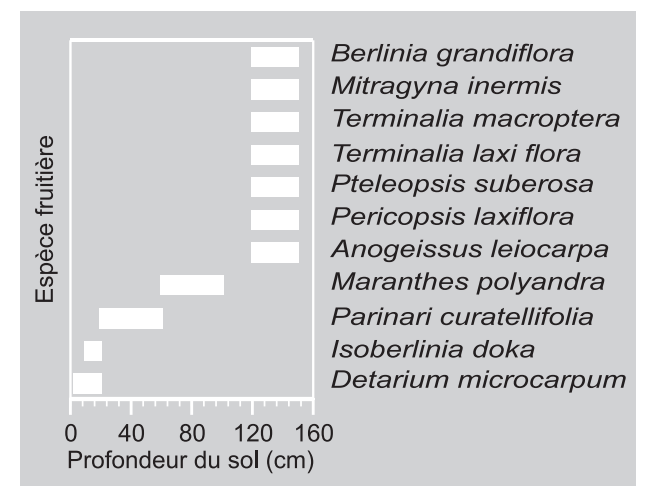

Figure 3.

Caractéristiques physicochimiques des sols dans les onze groupements végétaux identifiés de la forêt classée de Niangoloko (Burkina Faso).
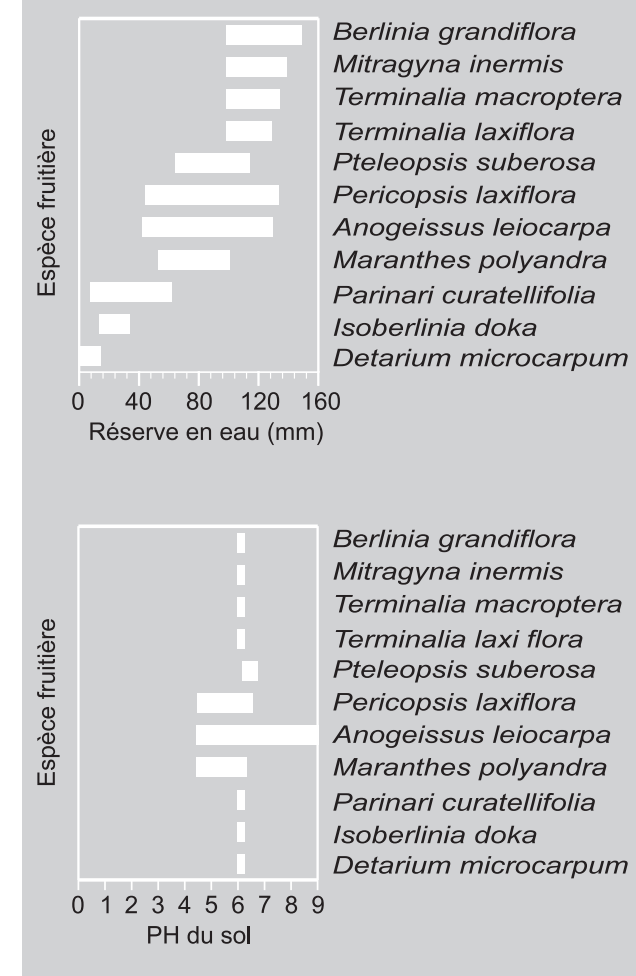

octobre ; ceux de Parinari curatellifolia, de septembre à novembre; enfin, les fruits de Maranthes polyandra sont disponibles de septembre à décembre. Ce décalage dans les dates de maturité des fruits pourrait permettre aux populations riveraines d'exploiter les arbres une grande partie de l'année.

\subsection{Utilisation des fruits des quatre espèces par les populations riveraines}

La pulpe de chacune des quatre espèces Detarium microcarpum, Flacourtia flavescens, 
Tableau III.

Période de récolte et productivité de quatre espèces fruitières trouvées en abondance dans la forêt classée de Niangoloko (Burkina Faso).

\begin{tabular}{lccc} 
Groupement & Période de disponibilité & \multicolumn{2}{c}{ Productivité } \\
\cline { 3 - 4 } & $\begin{array}{c}\text { Nombre moyen } \\
\text { de fruit par individu }\end{array}$ & $\begin{array}{c}\text { Poids moyen des fruits } \\
\text { produits par arbre } \\
\text { (kg en poids sec) }\end{array}$ \\
\hline Detarium microcarpum & Février-avril & $195 \pm 58$ & $2,1 \pm 0,6$ \\
Flacourtia flavescens & Juillet-octobre & $435 \pm 122$ & $0,5 \pm 0,2$ \\
Maranthes polyandra & Septembre-décembre & $130 \pm 64$ & $0,4 \pm 0,2$ \\
Parinari curatellifolia & Septembre-novembre & $168 \pm 53$ & $1,0 \pm 0,4$
\end{tabular}

Parinari curatellifolia et Maranthespolyandra est comestible. Les populations riveraines de la forêt consomment ces fruits occasionnellement, mais uniquement pour leur pulpe. Ils ne font l'objet d'aucun commerce. Des enquêtes réalisées auprès de ces consommateurs ont révélé qu'ils ignoraient que les espèces Maranthes polyandra (figure 4) et Parinari curatellifolia (figure 5) avaient des graines oléagineuses.

\subsection{Impact des activités des populations riveraines sur les écosystèmes}

Fruits de Maranthes polyandra : la pulpe est comestible et la graine oléagineuse peut intervenir dans la savonnerie.
Les populations riveraines de la forêt classée de Niangoloko exercent une intense pression sur ses écosystèmes par leurs activités

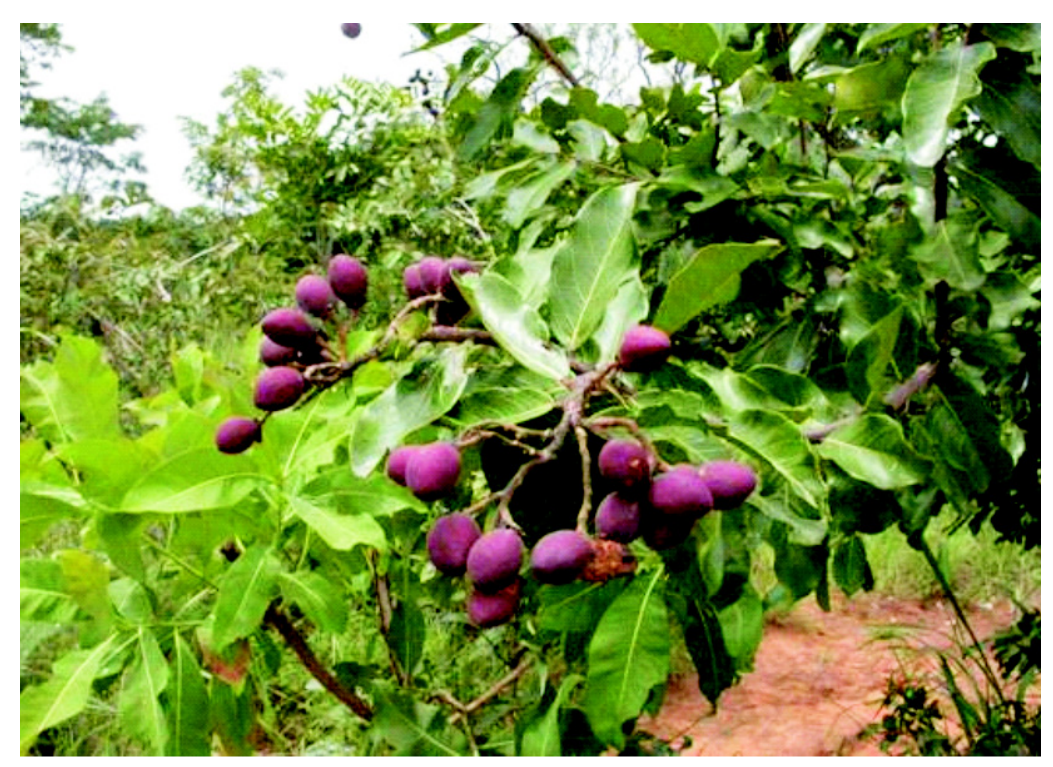

quotidiennes : pâturage, feux de brousse et coupe du bois utilisé comme source d'énergie (figure O). Paradoxalement on constate que parmi les quatre espèces fruitières dont les fruits pourraient faire l'objet d'une exploitation commerciale, Detarium microcarpum et Parinari curatellifolia sont les plus recherchées par les ménages pour leur exploitation en bois de chauffe, d'où l'observation de coupes intenses dans les peuplements de ces deux espèces (figure 6 ).

\section{Discussion}

Les fortes densités des arbres fruitiers de la forêt classée de Niangoloko pourraient justifier une exploitation commerciale de leurs fruits. En effet, pour Peter [9], de telles densités élevées conditionnent l'exploitation commerciale d'une ressource forestière car, à défaut, la faible représentation de l'espèce l'exposerait à une surexploitation et donc à son extinction. Par ailleurs, les espèces présentes en faibles densités sont peu faciles à localiser et leur récolte requière des coûts élevés en temps et en distances à parcourir ; elles ne permettent pas de bons rendements.

Les quatre espèces considérées dans notre étude, ayant été rencontrées dans la forêt à de fortes densités et sous forme de groupements végétaux, pourraient permettre de forts rendements tout en évitant leur surexploitation. L'accès à ces ressources est donc aisé pour des collecteurs pour qui la récolte est par ailleurs facilitée par la taille relativement petite de ces arbres fruitiers. 
Cela étant, une ressource forestière n'est rentable économiquement que si la mise en œuvre de son exploitation ne nécessite que de légers investissements et si la ressource exploitée a des usages multiples. Les quatre espèces considérées répondent à ces conditions, elles peuvent être facilement séchées au soleil [4] et ainsi être conservées pendant longtemps pour être écoulées progressivement.

Le véritable enjeu serait de pouvoir utiliser l'exploitation commerciale des ressources fruitières de la forêt classée de Niangoloko comme une stratégie de conservation de sa biodiversité. Durant la dernière décennie, une telle exploitation commerciale avait été suggérée comme une stratégie pouvant aider à réduire la déforestation dans les tropiques en général. L'idée en était que, si la valeur de la commercialisation des produits tirés des écosystèmes naturels étaient supérieurs aux retours escomptés de la destruction pour exploitation du bois de chauffe ou pour l'utilisation des terres pour l'agriculture, alors l'exploitation de la forêt pour ses productions fruitières pouvait être favorisée. Dans cette perspective, non seulement la biodiversité serait conservée incluant aussi bien les utilisations actuelles ou potentielles des plantes utiles, mais aussi d'autres services de la forêt comme la stabilisation du climat et l'attraction des touristes.

Les quatre espèces auxquelles nous nous sommes intéressés sont des espèces dont les fruits sont à haute valeur commerciale, ce qui est particulièrement vérifié pour les espèces oléagineuses Maranthes polyandra et Parinari curatellifolia : l'huile de Maranthes polyandra peut intervenir dans la cosmétique comme la fabrication de savon, quant à l'espèce Parinari curatellifolia, elle donne une huile utilisée pour l'alimentation, la peinture, les vernis et la fabrication d'encre [4]. Le potentiel fruitier pourrait alors être la source d'importants intérêts économiques et permettre d'apporter des revenus substantiels aux populations riveraines qui l'exploitent. De tels objectifs sont parfaitement réalistes ; il a été démontré dans plusieurs pays (Belize et Inde, par exemple) que des forêts bien gérées pour la cueillette des plantes médicinales rapportaient plus que si elles étaient défrichées pour la culture ou l'élevage [10].

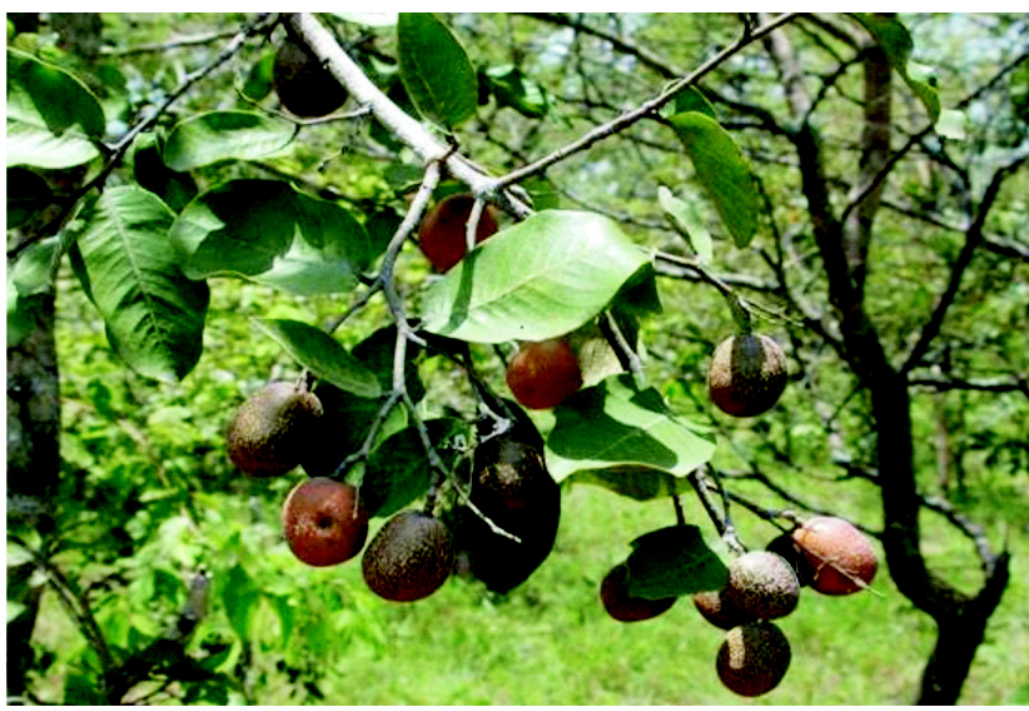

Figure 5. des quatre espèces fruitières Detarium microcarpum, Flacourtia flavescens, Parinari curatellifolia et Maranthes polyandra, la mise en valeur de leurs fruits pourra justifier qu'elles soient conservées et vulgarisées pour assurer leur survie. En effet, actuellement, seule Detarium microcarpum bénéficie du statut d'espèce intégralement protégée au Burkina Faso car elle fait partie des espèces en péril [7]. Les trois autres espèces, bien qu'étant économiquement importantes dans d'autres contrées comme

Fruits de Parinari curatellifolia : la pulpe est comestible et la graine oléagineuse intervient dans la cosmétique, le vernis, la savonnerie.

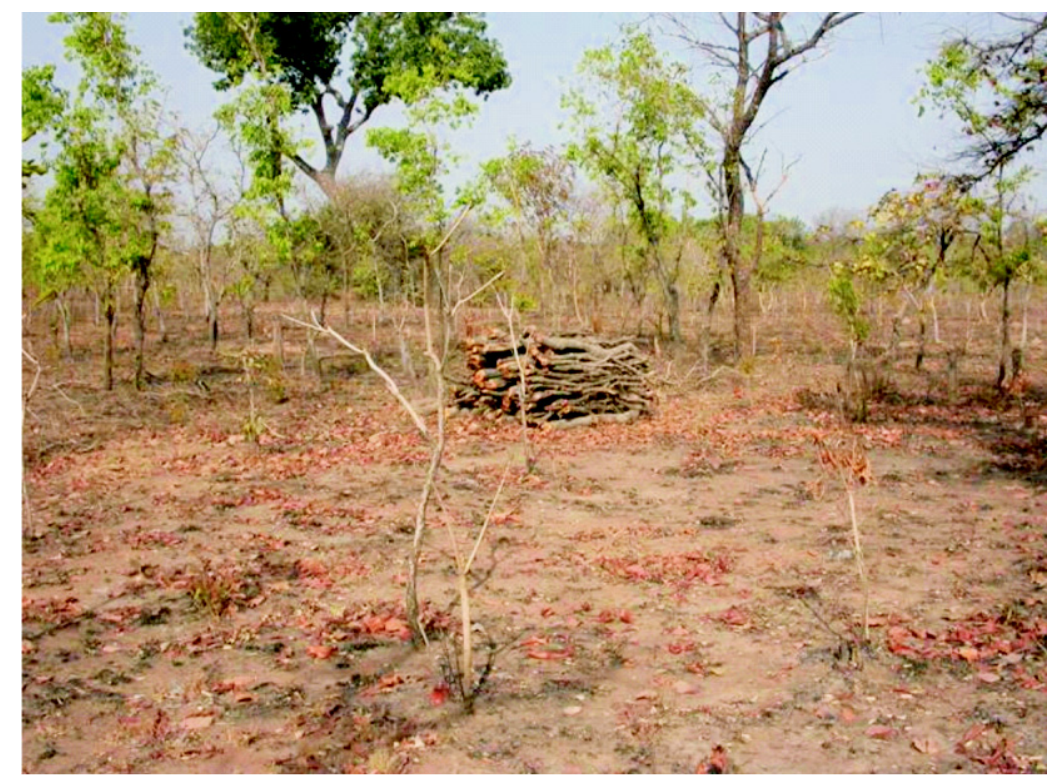


en Tanzanie et au Zimbabwe, sont méconnues au Burkina Faso où elles ne figurent pas sur la liste des espèces fruitières de première importance. Pourtant, ce sont des arbres à usages multiples puisqu'ils peuvent être exploités en agroforesterie [11]. Au Zimbabwé, Parinari curatellifolia est épargnée par les agriculteurs lors des défriches car, outre la production des fruits, elle participe à la fertilisation des sols et à la production de bois d'œuvre et de chauffe [12]. Cela corrobore bien les résultats obtenus lors de nos enquêtes : pour les populations riveraines de la forêt classée de Niangoloko également, le bois de Parinari curatellifolia fait partie (avec celui de Detarium microcarpum) des essences les plus recherchées pour le feu. De même, la présence des espèces Maranthes polyandra et Parinari curatellifolia leur permet de juger de l'état de fertilité d'un sol. Il y a donc un intérêt certain à préserver ces arbres lors des défriches, et même à les planter dans leurs champs. La plantation de Detarium microcarpum, Flacourtia flavescens, Parinari curatellifolia et Maranthes polyandra en terrain agricole ne poserait d'ailleurs aucun problème écologique majeur, ces quatre espèces supportant de larges variations de $\mathrm{pH}$, des sols peu profonds et des réserves en eau négligeables ; de telles caractéristiques sont des atouts importants car l'association de ces espèces à d'autres cultures ne génère aucun risque d'appauvrissement du sol. Enfin, la forêt classée de Niangoloko est aujourd'hui la seule au Burkina Faso à abriter d'importants peuplements à Flacourtia flavescens. Le risque est alors important de voir cette espèce disparaître du Burkina Faso. En Afrique du Sud, Flacourtia indica (Burm. F.) Merr., espèce très voisine de $F$. flavescens, est utilisée pour ériger des haies vives. Au Burkina Faso, la valorisation des fruits de $F$. flavescens, espèce épineuse se développant souvent en fourré dense, pourrait également amener les populations à utiliser cette espèce pour les haies vives tout en exploitant ses fruits.

\section{Conclusion}

Dans la forêt classée de Niangoloko, les conditions écologiques sont réunies pour une exploitation commerciale de certains fruits issus de cueillette. Le potentiel fruitier du site est très important et présente d'intéressantes perspectives financières pour les populations riveraines.

Si ces ressources naturelles étaient effectivement mises en valeur, elles pourraient être une opportunité pour les marchés de la sous-région et même d'ailleurs. Les revenus financiers récupérés alors par les familles vivant autour de cette forêt pourraient les aider à continuer à subvenir à leurs besoins sans avoir à couper ou à transformer cet espace hautement diversifié en domaine d'exploitation agricole. Les fruits de cette forêt sont d'une haute valeur commerciale et cela pourrait servir d'exemple pour d'autres sites classés de la région. Néanmoins, la population du Burkina Faso n'ayant pas de connaissance préalable sur l'exploitation industrielle des fruits des espèces présentes, il faudra envisager une phase préalable de formation des populations aux techniques de transformations avant qu'un bénéfice économique ne puisse être retiré.

\section{Remerciement}

L'étude a été financée par le programme de développement Enhancement of research capacity (Danish International Development Agency) (104. Dan.8.L. 203).

\section{Références}

[1] Lykke A.M., Local perceptions of vegetation change and priorities for conservation of woody-savannah vegetation in Senegal, J. Environ. Manage. 59 (2000) 107-120.

[2] Kambou S., Contribution à l'étude de la biologie florale et de la régénération de Anogeissus leiocarpus (DC.) Guill. et Perr. au Burkina Faso, Univ. Ouagadougou, Ouagadougou, Burkina Faso, Mém. DEA, 1992, 124 p.

[3] Ramade F., Conférence introductive : intérêt des aires protégées pour la recherche en écologie de la conservation: des fondements aux applications, CR Biol. 326 (2003) 3-8.

[4] Hines D.A., Eckman K., Indigenous multipurpose trees for Tanzania: uses and economic benefits for people, FO:Misc/93/9, working pap., FAO, Rome, Italy, 1993. 
[5] Arbonnier M., Arbres, arbustes et lianes des zones sèches d'Afrique de l'Ouest, CIRADMNHN-UICN, La librairie du Cirad, Montpellier, France, 2000.

[6] Anon., Étude morpho-pédologique des forêts classées du Houet et de la Comoé, Projet Carto, Bureau National des Sols (Bunasol), Ouagadougou, Burkina Faso, 1996, 65 p.

[7] Anon., Monographie nationale sur la diversité biologique du Burkina Faso, Conages, Ouagadougou, Burkina Faso, 1999.

[8] McCune B., Grace J.B., Analysis of ecological communities, MjM Software Design, Gleneden Beach, USA, 2002.

[9] Peters C.M., Sustainable harvest of non-timber plant resources in tropical moist forest: an ecological primer, Biodiversity Support Pro- gramme, World Wildlife Fund, Washington DC, USA, 1994.

[10] Balick M.J., Cox P.A., Ethnobotanical research and traditional health care in developping countries, in: Bodeker G., Bhat K.K.S., Burley J., Vantomme P. (Eds.), Nonwood forest product, Vol. 11, FAO, Rome, Italy, 1997.

[11] Wood P. J., Burley J., Les arbres à usages multiples. Introduction et évaluation pour l'agroforesterie, Centre Technique de Coopération Agricole et Rurale ACP-CEE (CTA), (Ed. fr.), Wageningen, Pays-Bas, 1993.

[12] Packham J., The value of indigenous fruitbearing trees in Miombo woodland areas of south-central Africa, Overseas Development Institute, Social Forestry Network Pap., 15c London, UK, 1993, pp. 13-20.

\section{El potencial frutero de la selva clasificada desde Niangoloko hasta Burkina} Faso.

Resumen - Introducción. El objeto de nuestro estudio fue promover en la selva clasificada de Niangoloko unas actividades adicionales, menos destructoras que la exploatación de la madera en los ecosistemas. Cuatro especies fruteras (Detarium microcarpum Guill. \& Perr., Flacourtia flavescens Willd., Maranthes polyandra Benth. y Parinari curatellifolia Planch. ex Benth.) se representan aquí abundantemente. La pulpa de cada una de estas frutas es comestible y se puede transformar en mermelada. Las semillas de Parinari curatellifolia y Maranthes polyandra son oleoginosas y se utilizan en numerosos sectores de la industria. Desafortunadamente, bien que estas frutas se utilizan en la industria de otros países africanos, se desconocen en las poblaciones del Burkina Faso. Por esta razón, con el fin de valorar mejor estos recursos, nuestro estudio procuró constituir una base de datos sobre la localización de las poblaciones de especies fruteras, su ecología y su potencial de producción en frutas. Material y métodos. Con la ayuda de un mapa pedológico y de un GPS, pudo realizarse un mapa de agrupaciones vegetales. Unas muestras permitieron medir la densidad y la altura de los árboles de las especies fruteras en cada agrupación. La producción media de frutas por árbol para cada especie también se midió. Resultados. Se seleccionaron y cartografiaron once agrupaciones vegetales. Cada especie frutera tiene una localización bien precisa en una o varias agrupaciones. Se seleccionaron poblaciones de Detarium microcarpum, Flacourtia flavescens, Maranthes polyandra et Parinari curatellifolia con densidades respectivas pudiendo alcanzar $(1486 \pm 110 ; 160 \pm 33 ; 313 \pm 32$ y $392 \pm 39)$ individuos $\cdot \mathrm{ha}^{-1}$. La cantidad de frutas producida por individuo fue respectivamente de $(2,1 \pm 0,6 ; 0,5 \pm 0,2 ; 0,4 \pm 0,2 \mathrm{y}$ $1,0 \pm 0,4) \mathrm{kg}$ de frutos secos. La altura media de los árboles frutales apenas sobrepasa $(3,7 \pm$ $0,3) \mathrm{m}$, esto permite una colecta fácil de las frutas sin riesgo de accidentes. Conclusión. Las condiciones ecológicas permiten una explotación comercial de las frutas. El potencial frutero del bosque clasificado desde Niangoloko es muy importante y presenta unas perspectivas financieras interesantes para las poblaciones ribereñas.

Burkina Faso / Detarium microcarpum / Flacourtia flavescens / Marantbes polyandra / Parinari curatellifolia / bosques / rodales / frutales / inventarios forestales / ordenación de recursos 